\title{
Characterization of the role of the photosensitizer, deuteporfin, in the detection of lymphatic metastases in a pancreatic cancer xenograft model
}

\author{
XINZHE YU* , HENGCHAO LI*, DELIANG FU, CHEN JIN and JI LI \\ Department of Pancreatic Surgery, Huashan Hospital, Fudan University, Shanghai 200040, P.R. China
}

Received September 18, 2014; Accepted June 5, 2015

DOI: $10.3892 / \mathrm{ol} .2015 .3441$

\begin{abstract}
Currently, the use of photosensitizers as tracer agents to detect lymphatic metastases is a developing area of study in the field of pancreatic cancer treatment. In the present study, deuteporfin, a novel photosensitizer, was used as a tracer agent to detect lymphatic metastases in a pancreatic cancer xenograft model. The biodistribution and pharmacokinetics of deuteporfin, following intravenous administration and injection of deuteporfin into the left rear footpad, were investigated in Sprague-Dawley rats. The increased difference in deuteporfin concentration between the cancerous and normal tissues was directly observed through the application of a Wood's lamp. In addition, the highly lymphatic BxPC-3-LN5 human metastatic pancreatic cancer cell line was generated from BxPC-3 cells using a continuous screening and seeding method in vivo. A xenograft model of the BxPC-3-LN5 human pancreatic cancer cell line transplanted into the left rear footpad of nude mice, was established. The effects of deuteporfin as a tracer agent in the detection of lymphatic metastases were then characterized in the pancreatic cancer xenograft model. Following intravenous administration, deuteporfin was rapidly enriched in the pancreas and popliteal fossa lymph nodes compared with that of the left rear footpad administration group. In addition, deuteporfin appeared to be selectively enriched in the cancerous pancreatic lymph nodes of the pancreatic cancer xenograft model. These results indicated that deuteporfin may be developed as a novel photosensitizer tracer agent for the detection of lymphatic metastases in pancreatic cancer. The advantages of deuteporfin are that it has a selective tumor-targeting effect due to high tissue uptake, and that it may be administered intravenously and is therefore suitable for surgery.
\end{abstract}

Correspondence to: Professor $\mathrm{Ji} \mathrm{Li}$, Department of Pancreatic Surgery, Huashan Hospital, Fudan University, 12 Middle Urumqi Road, Shanghai 200040, P.R. China

E-mail: liji.surgeon@gmail.com

*Contributed equally

Key words: lymphatic metastases, photosensitizer, tracer agent, photodynamic therapy

\section{Introduction}

Pancreatic cancer represents the fourth most common cause of cancer-associated mortality in the western world $(1,2)$. Despite significant progress in basic and clinical research, pancreatic cancer remains a significant therapeutic challenge to humans, with 1- and 5-year survival rates of just 20 and 6\%, respectively $(3,4)$. The difficult surgical approach, resistance to conventional therapies and subsequent low survival rates all contribute to the high frequency of occurrence of lymphatic metastases (5-7). Currently, the use of tracer agents in the detection of lymphatic metastases is a focus of attention, as it facilitates specification of treatment options for the given diagnosis. However, at present, there is no acceptable and ideal imaging modality or technique for the accurate detection of lymphatic metastases $(8,9)$. For example, the use of isotope tracing is limited due to the potential side-effects of irradiation, as well as the vague surgical resolution provided by the blue dye $(10,11)$. Computed tomography and conventional magnetic resonance imaging are not sufficiently reliable for the accurate detection of lymphatic metastases. The limitations of these tracing methods are due to the following two aspects: i) They possess no tumor targeting effect; and ii) the route of administration is typically through the lymphatic system, which is difficult for the treatment of pancreatic cancer as the pancreas is a retroperitoneal organ (12). Therefore, the development of a novel lymph node tracer and imaging method is required.

Photodynamic therapy (PDT), based on the photogeneration of highly cytotoxic singlet oxygen species and the subsequent induction of oxidative stress that results in the death of stained cells, is currently used in oncology (13). PDT is a minimally invasive treatment that damages target cells by inducing cytotoxicity via the generation of cytotoxic oxygen species. The PDT components, comprising the photosensitizer, light and oxygen, are individually non-toxic. However, exposure of the photosensitizer to light in the presence of oxygen induces the generation of highly reactive singlet oxygen $\left({ }^{1} \mathrm{O}_{2}\right)$ species within the tumor tissue, which results in significant damage of the cells in proximity to the treated area (14-18). PDT is a widely accepted treatment strategy for numerous types of cancerous and precancerous lesion, including those in the bladder, brain, ovary and pancreas (19-21). However, most attention has been directed towards the therapeutic role of the photosensitizer, rather than its potential function as a tracer agent. 
Deuteporfin, one of the novel generation of photosensitizers, is particularly photosensitive to light with wavelengths of 400-700 nm. Chemically, the structure of deuteporfin is composed of four distinct sub-porphyrin derivatives: Methoxyethy-hydroxyethyl-dipropionic, di-methoxyethyl-dipropionic, hydroxyethy-vinyl-dipropionic and methoxyethy-vinyl-dipropionic (Fig. 1). Compared with traditional lymph node tracers, deuteporfin has a significant advantage in terms of two aspects: i) Selective targeting of tumors due to high tissue uptake and ii) that it may be administered intravenously. The present study aimed to exploit these features of deuteporfin for use as a tracer agent, in order to detect lymphatic metastases in a pancreatic cancer xenograft model. The study was approved by the ethics committee of Fudan University, Shanghai, China.

\section{Materials and methods}

Reagents. Deuteporfin was obtained from the R\&D Center of Fudan-Zhangjiang Bio-Pharmaceutical Co. Ltd. (Shanghai, China) and was used without further purification. Normal saline was purchased from Shanghai Baxter Healthcare Co., Ltd., Shanghai, China. Absolute ethanol, high glucose-Dulbecco's modified Eagle's medium (H-DMEM), fetal bovine serum (FBS), trypsin, EDTA, formalin, paraffin, and hematoxylin and eosin (H\&E) were purchased from Sinopharm Chemical Reagent Co., Ltd. (Shanghai, China). In addition, deionized water was from Fudan University (Shanghai, China), and Tris-buffered saline and phosphate-buffered saline were prepared in our laboratory. All solvents and chemicals were of analytical grade.

Experimental animals. Sprague-Dawley (SD) rats and BALB/C-nu/nu nude mice were purchased from the Shanghai Laboratory Animal Co. Ltd. (Shanghai, China). The SD rats and BALB/c-nu/nu male mice were housed in a pathogen-free animal facility. The temperature was maintained at $23^{\circ} \mathrm{C}$ with a humidity of $50-60 \%$, and the mice were subjected to a 10/14-h light/dark cycle. All mice were provided with food and water 3 times a week by professional technicians. All animal studies were in compliance with the approved animal protocols and the guidelines of the Institutional Animal Care and Use Committee of Fudan University. The BxPC-3 human pancreatic cancer cell line was obtained from the Shanghai Branch of the Chinese Academy of Science, Shanghai, China.

Cell culture. The highly lymphatic metastatic pancreatic cancer cell line was generated from BxPC-3 cells by a continuous screening and seeding method in vivo. Briefly, $1 \times 10^{7}$ BxPC-3 cells in $100 \mu 1$ normal saline were subcutaneously injected into the left rear footpad of male BALB/C-nu/nu nude mice ( $n=5$; aged $6-8$ weeks). Six weeks later, the mice were anesthetized by intravenous injection with chloral hydrate and sacrificed by cervical dislocation. The lymph nodes located in the left knee, groin, side of the iliac artery and porta renis (depression in the kidney) were then collected. All the conjunctive tissues were removed and the lymph nodes were gently crushed on a 200-mesh sieve (Sinopharm Chemical Reagent Co., Ltd.). The cells routinely cultured in H-DMEM containing $10 \% \mathrm{FBS}$ in an incubator at a temperature of $37^{\circ} \mathrm{C}$ for 2-3 days. Subsequently, cells were digested by incubation

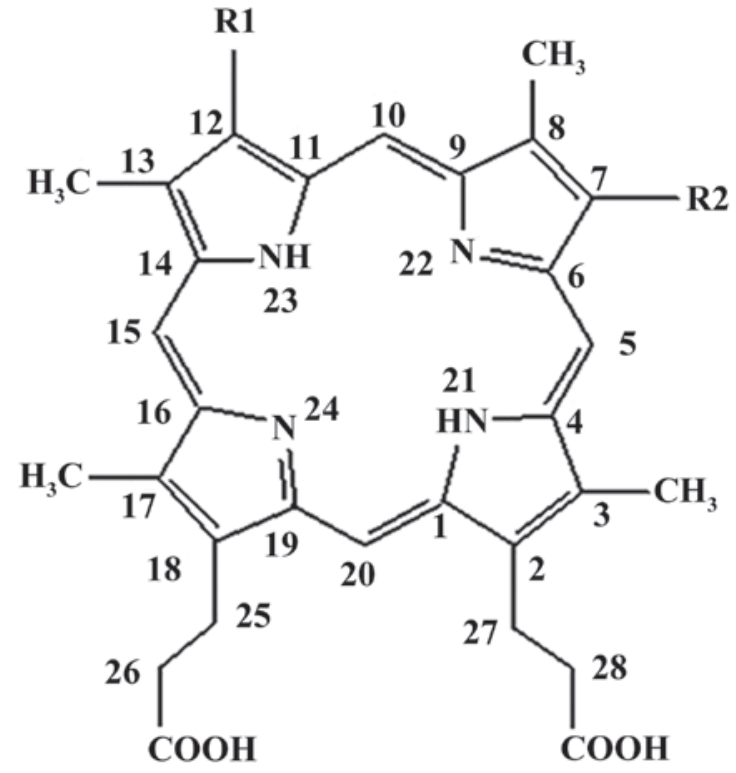

Figure 1. Chemical structure of deuteporfin. $\mathrm{R} 1=\mathrm{CH}_{3} \mathrm{CH}\left(\mathrm{OCH}_{3}\right)$ and $\mathrm{R} 2=\mathrm{CH}_{3} \mathrm{CHOH}$ or $\mathrm{R} 1=\mathrm{CH}_{3} \mathrm{CHOH}$ and $\mathrm{R} 2=\mathrm{CH}_{3} \mathrm{CH}\left(\mathrm{OCH}_{3}\right)$ or $\mathrm{R} 1=\mathrm{CH}_{3} \mathrm{CH}$ $\left(\mathrm{OCH}_{3}\right)$ and $\mathrm{R} 2=\mathrm{CH}_{3} \mathrm{CH}\left(\mathrm{OCH}_{3}\right)$.

with $0.025 \%$ trypsin $/ 0.002 \%$ EDTA and then reinoculated into another group of mice. This procedure was repeated 5 times (total mice, $\mathrm{n}=25$ ), until the BxPC-3-LN5 subline was obtained, comprising BxPC-3 cells with an increased capacity to metastasize.

Pharmacokinetics and tissue distribution of deuteporfin in vivo. Seventy-two SD rats were randomly divided into two equal groups, comprising the caudal vein administration group $(n=36)$ and the left rear footpad administration group $(\mathrm{n}=36)$. The rats were injected with $2.5 \mathrm{mg} / \mathrm{ml}$ deuteporfin either intravenously or in the left rear footpad. At various time-points (1, 3, 6, 12, 24 and $48 \mathrm{~h})$ post-injection, the rats were sacrificed by cervical dislocation and blood samples were obtained from the aorta abdominalis ( $n=6$ rats per timepoint). The tissues of interest (including the heart, liver, spleen, lung, kidney, pancreas, popliteal lymph nodes and dorsal skin) were collected immediately, weighed and homogenized with an adequate quantity of physiological saline. Following centrifugation at $644 \mathrm{x} g$ at $25-26^{\circ} \mathrm{C}$ for $5 \mathrm{~min}$, the homogenate was filtered and the filtrate was boiled, cooled and centrifuged again $\left(644 \mathrm{x} \mathrm{g}\right.$ at $25-26^{\circ} \mathrm{C}$ for $\left.5 \mathrm{~min}\right)$. The deuteporfin content of each tissue of interest was determined by the fluorometric method, using a fluorescence spectrometer (F-4500; Hitachi High-Technologies Corp., Tokyo, Japan) at excitation and emission wavelengths of 295 and $610 \mathrm{~nm}$, respectively. The standard curve of deuteporfin was obtained by plotting the concentration vs. fluorescence intensity.

Generation of popliteal fossa lymphatic metastasis model. The popliteal fossa (depression behind the knee joint) lymphatic metastases model was generated as a xenograft of the BxPC-3-LN5 human cancer cell line. Briefly, $\sim 1 \times 10^{7}$ BxPC-3-LN5 cells in $100 \mu 1$ normal saline were injected subcutaneously into the left rear footpad of thymus-deficient 8-week-old BALB/C nude mice ( $\mathrm{n}=5$; weighing 18-20 g). 
Five weeks later, a lymph node intumescence or swelling that was $3-5 \mathrm{~mm}$ in diameter was able to be observed in the left knee. This procedure was repeated five times (total mice, $\mathrm{n}=25)$. The mice were sacrificed by cervical dislocation and the intumescent lymph nodes were removed and fixed in $10 \%$ neutral buffered formalin. The specimens were dehydrated in a graded series of alcohol, and embedded in paraffin. Serial $5-\mu \mathrm{m}$ sections were deparaffinized, hydrated and stained with $\mathrm{H} \& \mathrm{E}$ in order to detect pancreatic cancer lymphatic metastases.

Localization of deuteporfin in tumors at inoculation site and sites of lymphatic metastases. The BALB/C-nu/nu nude mice with pancreatic cancer metastases were intravenously injected with deuteporfin at a dose of $2.5 \mathrm{mg} / \mathrm{ml}$. At given time intervals $(1,2,4,6,12,24$ and $48 \mathrm{~h})$ following the injection, the mice were sacrificed by cervical dislocation. The lymph nodes in the left (metastatic side) and right (control side) popliteal fossa, respectively, were collected immediately. The deuteporfin content was measured according to the aforementioned fluorescence method.

Wood's lamp observations. The difference in deuteporfin concentration between the cancerous and normal tissues was directly observed using a Wood's lamp (model, BS-WL1) purchased from the Sincery International Ltd. (Shanghai, China). Briefly, the light was turned off and the cover shade was secured prior to usage. The Wood's lamp was placed parallel to the mice at a distance of $15-20 \mathrm{~cm}$ and then tuened on. Characteristics of the mice were identified by the appearance of different colors under the ultraviolet ray.

Statistical analysis. The number of independent replicates is listed individually for each experiment. All data are expressed as the mean \pm standard deviation. Statistical analyses were performed with analysis of variance using SPSS 20.0 software (IBM SPSS, Armonk, NY, USA) and $\mathrm{P}<0.05$ was considered to indicate a statistically significant difference.

\section{Results}

Intravenous administration of deuteporfin is advantageous over footpad administration. The efficiency of intravenous administration of deuteporfin was compared with that of administration via the left rear footpad. In general, the injected SD rats appeared to be healthy throughout the experimental period and no symptoms of a toxic reaction to light, for example partially inflamed skin or abscesses, were observed. Furthermore, deuteporfin was observed to be distributed in all tissues of the body following intravenous or left rear footpad administration. However, the distribution of deuteporfin was uneven due to differences in blood perfusion and the tissue microenvironment.

The mean deuteporfin levels in the blood and tissues following intravenous administration are exhibited in Table IA. It was observed that deuteporfin levels following intravenous treatment decreased as follows: Blood $>$ liver $>$ kidney $>$ lymph node $>$ pancreas $>$ skin $>$ heart $>$ lungs $>$ spleen. The time-course changes of deuteporfin levels in the blood, liver, kidney, heart, lungs and spleen were similar, with the highest concentration at $1 \mathrm{~h}$ post-injection, followed by a time-dependent decrease. The highest expression values attained were 108.31 \pm 1.02 , $75.18 \pm 3.57,58.52 \pm 5.19,13.01 \pm 2.10,1.89 \pm 2.48$ and $0.36 \pm 0.57 \mu \mathrm{g} / \mathrm{l}$, respectively. Notably, the normal liver exhibited a higher level of accumulation of deuteporfin. Previously, evidence of the poor sensitivity of cancerous liver tissue to photosensitization was observed when compared with that of the normal liver tissue (22). Therefore, caution must be taken when using PDT in the treatment of hepatic disease.

The mean levels of deuteporfin in the blood and tissues following left rear footpad administration are displayed in Table IB. In comparison to the intravenous administration group, the entry rates of deuteporfin into the tissues were markedly slower. For example, deuteporfin levels in the liver, kidney, lymph node and blood were demonstrated to reach a peak level at $3 \mathrm{~h}$ post-injection, and reached levels of $66.81 \pm 3.01$, $82.25 \pm 15.60,71.75 \pm 15.68$ and $73.26 \pm 2.87 \mu \mathrm{g} / 1$, respectively. However, the deuteporfin levels in the heart, spleen, lungs and pancreas peaked at $1 \mathrm{~h}$ post-injection and were subsequently eliminated with time. Additionally, the spleen and lungs exhibited their removal or resisting effects to deuteporfin, as the highest deuteporfin concentrations observed were only $1.11 \pm 1.42$ and $2.93 \pm 0.88 \mu \mathrm{g} / 1$, respectively. As for the skin, a higher level of deuteporfin $(30.57 \mu \mathrm{g} / \mathrm{l})$ was observed at $1 \mathrm{~h}$ post-injection and the peak concentration was detected at $12 \mathrm{~h}$ post-injection. It is notable that, following footpad administration, deuteporfin may directly enter the popliteal lymph nodes along the lymphatic vessels.

Deuteporfin may be used as a tracer to detect lymphatic metastases in a pancreatic cancer xenograft model. Regional lymphatic metastases from pancreatic cancer is considered to be relevant to tumor spread and progress (23). Therefore, a reliable lymphatic metastases model of pancreatic cancer that effectively mimics human pancreatic cancer and may be used to test the efficacy of therapeutic strategies, including resection and tracing, is urgently required (24-26). Xenografts of the BxPC-3-LN5 pancreatic cancer cell line with lymphatic metastases were generated in athymic nude mice. Tumors developed at the site of direct implantation of BxPC-3-LN5 cells in all the animals (Fig. 2A). The incidence of lymphatic metastases was $100 \%$ and the metastatic growth of tumor cells was observed in all cases. Swelling of the popliteal fossa lymph node was also observed 5 weeks following implantation on the metastatic side, but not on the control side (Fig. 2B). Immunohistochemical evaluation revealed an aggressive invasion of cancer cells in the lymph nodes. Initially, cancer cells were able to enter the marginal sinus and form isolated tumor cells or small groups of metastatic niduses (Fig. 2C). Subsequently, tumor cells accumulated to form large metastatic niduses. Certain tumor cells were also able to infiltrate the intermediate sinuses and proliferate, diffuse toward medullary sinuses and invade the whole lymph node (Fig. 2D).

Based on this lymphatic metastases model of pancreatic cancer, the extent of accumulation of deuteporfin in the bilateral popliteal fossa lymph nodes, following intravenous administration, was determined. As shown in Fig. 3, the two popliteal fossa lymph nodes exhibited similar metabolic kinetic trends, and deuteporfin accumulated with the highest concentration at $6 \mathrm{~h}$ following administration. However, the 


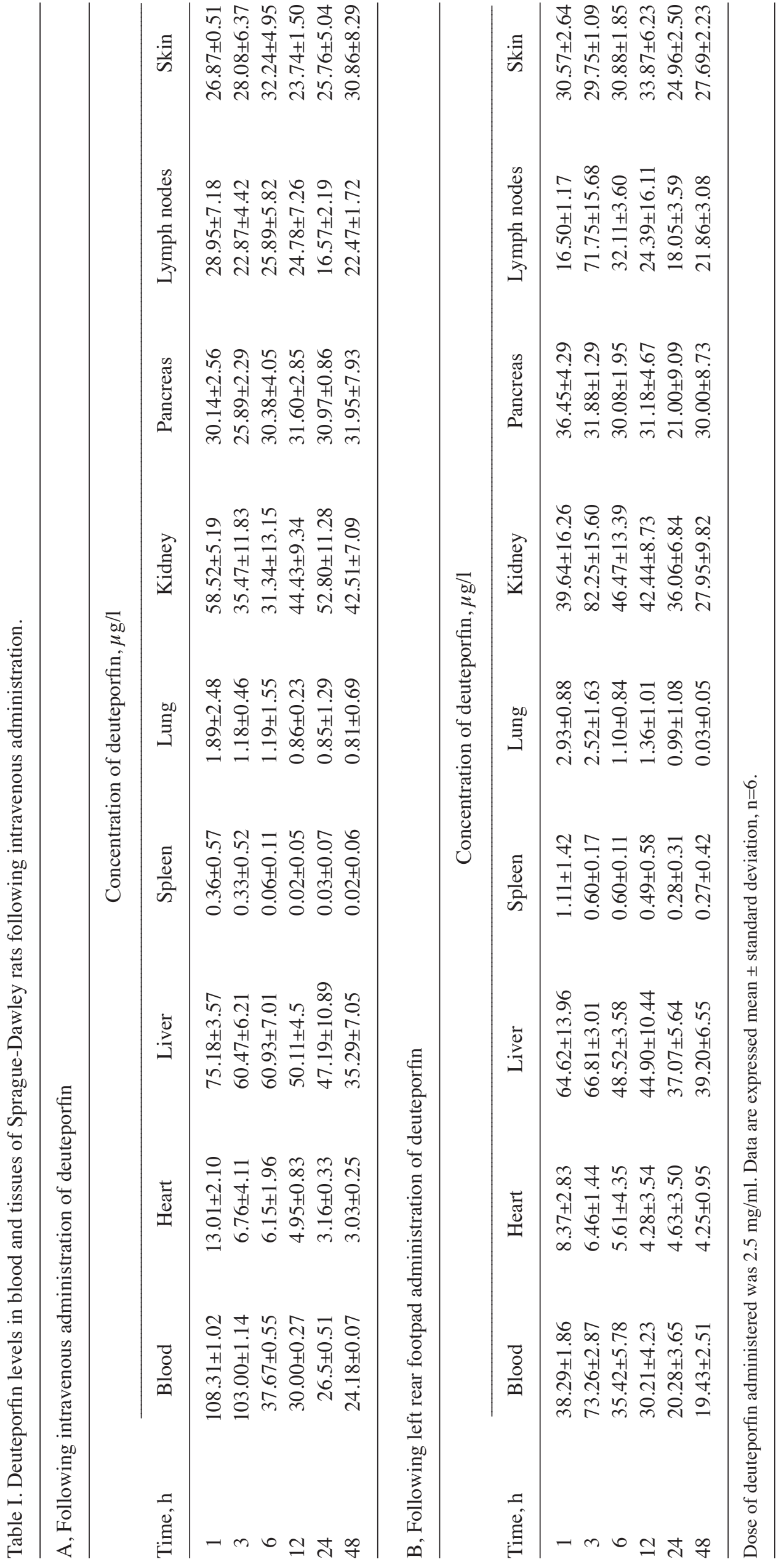




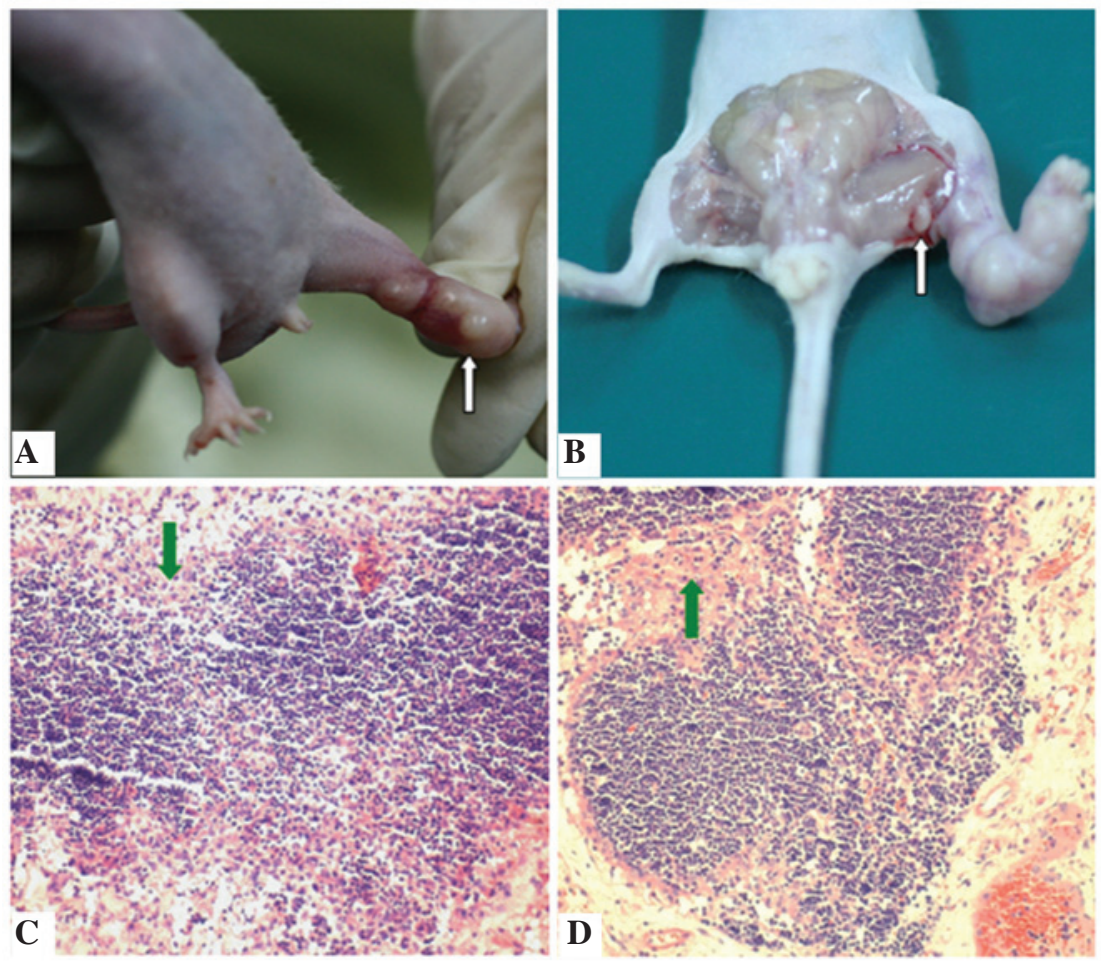

Figure 2. Pathological changes observed in a xenograft model of lymphatic metastatic pancreatic cancer cell line BxPC-3-LN5. (A) Primary tumors at the site of direct implantation of BxPC-3-LN5 cells in nude mice (white arrow). (B) Lymph node metastases (white arrow). (C) Immunohistochemical evaluation of cancer cell invasion to lymph nodes at the marginal sinus (green arrows). (D) Immunohistochemical evaluation indicated that metastatic cancer cells were able to grow in regional lymph nodes (green arrows). Hematoxylin and eosin staining; magnification, x200.

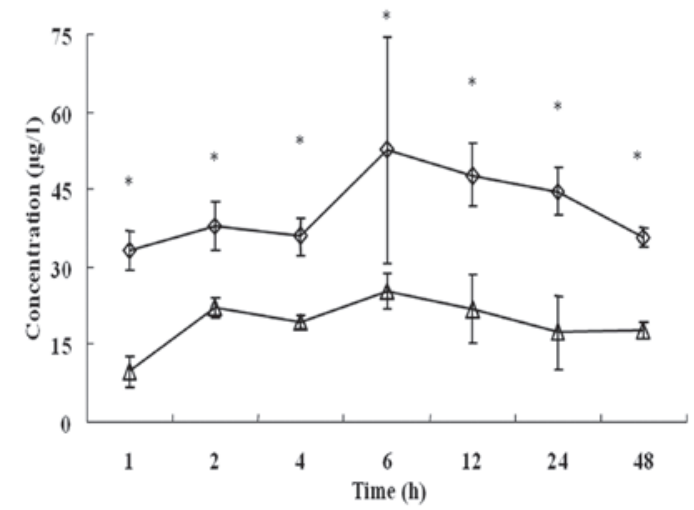

Figure 3. Time-course changes in deuteporfin levels in the popliteal fossa lymph nodes of the metastatic side $(\diamond)$ and the untreated side $(\Delta)$ of the human pancreatic cancer BxPC-3-LN5 cell xenograft mouse model. Values are expressed as the mean \pm standard deviation. ${ }^{*} \mathrm{P}<0.05$.

deuteporfin concentration in the lymph nodes was $\sim 2-3$ times higher on the metastatic side than that of the control side $(\mathrm{P}<0.05)$. Furthermore, the concentration of deuteporfin reached $33.10 \pm 3.81 \mu \mathrm{g} / 1$ at $1 \mathrm{~h}$ and $52.59 \pm 21.91 \mu \mathrm{g} / 1$ at $6 \mathrm{~h}$, respectively, on the metastatic side; while the concentration of deuteporfin on the control side was $9.72 \pm 2.95 \mu \mathrm{g} / 1$ at $1 \mathrm{~h}$ and $25.13 \pm 3.43 \mu \mathrm{g} / \mathrm{l}$ at $6 \mathrm{~h}$, respectively. The deuteporfin concentation peaked at $24 \mathrm{~h}$ on the metastatic and control sides. Subsequently, the concentration of deuteporfin in the bilateral popliteal fossa lymph nodes gradually decreased and reached $17.64 \pm 1.56$ and $35.58 \pm 1.95 \mu \mathrm{g} / \mathrm{l}$ at $48 \mathrm{~h}$ post-injection, respectively. A previous study reported that pancreatic cancer cells are selective to the uptake of photosensitizers, and that the ratio of uptake of deuteporfin between pancreatic cancer cells and normal tissues is 3:1 (27). Furthermore, the selectivity of cancer tissues for the photosensitizer is likely explained by their characteristic abnormal physiology, including poor lymphatic drainage, leaky vasculature, lower $\mathrm{pH}$ environment, higher level of receptors for low-density lipoproteins and abnormal stromal composition $(19,28,29)$. In the present study, the concentration of deuteporfin in the bilateral popliteal fossa lymph nodes of normal nude mice was also evaluated, and reached $28.19 \pm 1.71$ and $26.16 \pm 1.5 \mu \mathrm{g} / 1$, respectively, $24 \mathrm{~h}$ post-injection. This result indicated that the selectivity of cancer tissue to deuteporfin led to a significant decrease in the deuteporfin concentration in the corresponding normal tissue $(\mathrm{P}=0.002)$. The increased difference in drug concentration between the cancerous and normal tissues was directly observed through the application of the Wood's lamp (Fig. 4).

\section{Discussion}

PDT, through the use of an intravenously administered photosensitizer, has been used as an anti-vascular and anti-tumor therapeutic strategy for several decades (30). Furthermore, the development of lymph node-specific PDT has generated notable interest. Kilarski et al (31) reported that anti-lymph node PDT allows the control of lymphatic ablation and regeneration by alteration of the light fluence and photosensitizer dose. Other potential therapeutic uses of lymph node-specific PDT include the inhibition of the spread of lymph node-trafficking parasites or pathogens, as well 

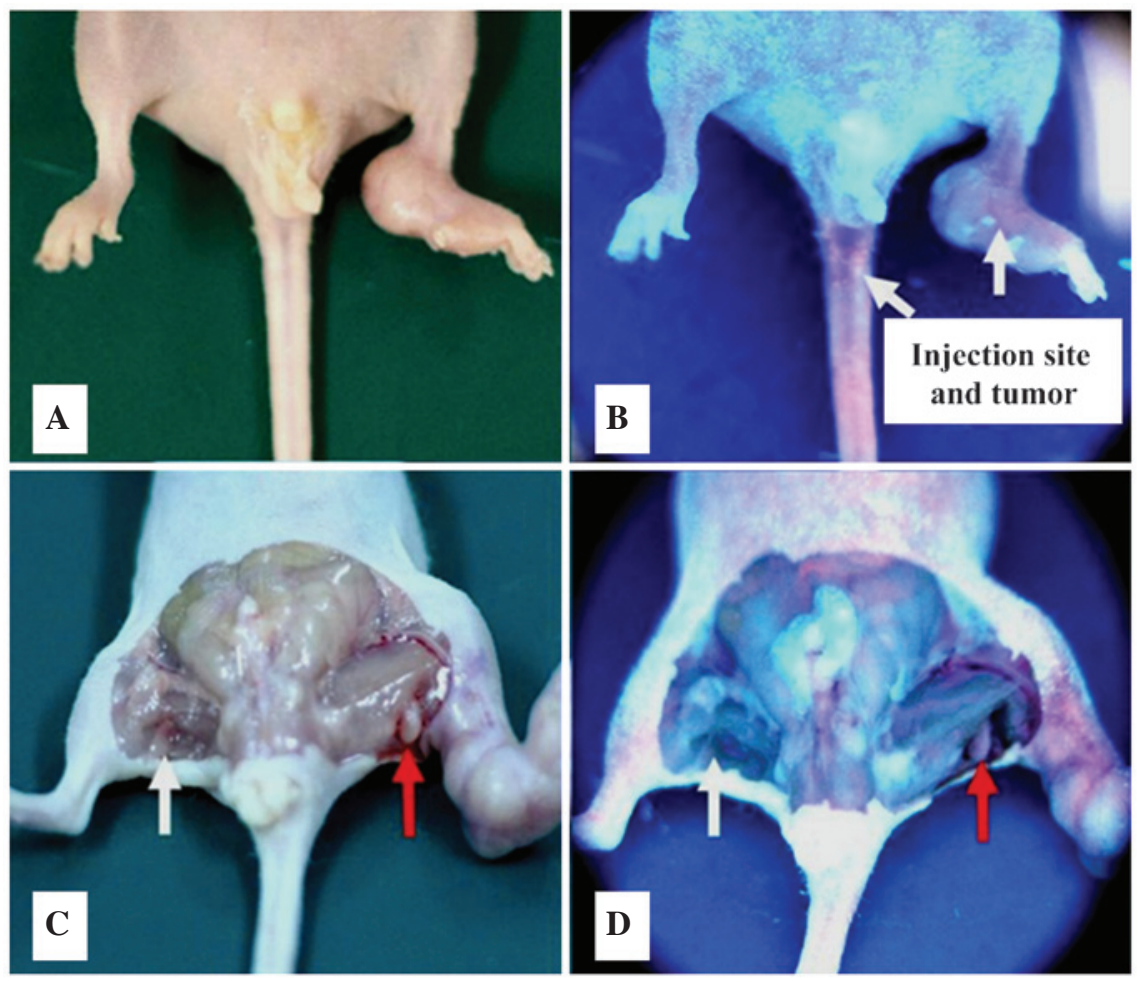

Figure 4. Tracking of deuteporfin to the tumor sites of the lymph node metastatic model of a pancreatic cancer cell line under irradiation of a Wood's lamp. Mice $1 \mathrm{~h}$ after intravenous administration of deuteporfin (A) without and (B) with the Wood's lamp. Excised mice $1 \mathrm{~h}$ after intravenous administration of deuteporfin (C) without and (D) with the Wood's lamp. White arrows indicate the normal popliteal fossa lymph nodes and the red arrows indicate the metastatic lymph nodes. Blue fluorescence indicates the effect of the Wood's Lamp.

as slowing the clearance of locally delivered drugs $(32,33)$. However, the application of a photosensitizer as a tracing agent for the detection of lymphatic metastases of pancreatic cancer has not previously been reported or fully explored, to the best of our knowledge.

Deuteporfin, one of the latest generation of photosensitizers, is characterized by clear and relatively pure chemical constituents, and has been demonstrated to possess good photodynamic anti-tumor activity (34); however, its role as a photosensitizer has not previously been studied. The present study aimed to elucidate the preclinical pharmacokinetic characteristics of deuteporfin, as well as using it in the detection of lymphatic metastases in a pancreatic cancer xenograft model. Similarly to previous reports (34), deuteporfin was rapidly eliminated throughout the mouse model, which suggested that it may be a promising drug with few side effects.

The current study was divided into two sections. The first section aimed to identify an appropriate route of administration for deuteporfin. The biodistribution and pharmacokinetics of deuteporfin in SD rats, following administration via separate routes, were therefore investigated. In the second section, a lymphatic metastatic pancreatic cancer xenograft model was established, and the biodistribution of deuteporfin in cancerous and normal lymph nodes was subsequently compared. It was demonstrated that the concentration of deuteporfin in the metastatic lymph nodes was highest $6 \mathrm{~h}$ following administration, and was $\sim 2-3$ times higher than that in the normal lymph nodes.

Following intravenous administration, only $1.89 \pm 2.48$ and $0.36 \pm 0.57 \mu \mathrm{g} / \mathrm{l}$ of deuteporfin were detected in the lungs and spleen, respectively, $1 \mathrm{~h}$ post-injection. These low values may be a result of specific removal or resistance mechanisms of these organs to xenenthesis (the introduction of a novel drug in the organ). Notably, the metabolic and retention effects of deuteporfin in the lymph nodes, skin and pancreas stabilized, and deuteporfin reached relatively stable levels in these organs at $48 \mathrm{~h}$ post-injection. These characteristics of deuteporfin distribution indicate that it may be helpful in the detection of lymphatic metastases and in the treatment of pancreatic cancer.

By contrast, the lymph node deuteporfin concentration in the footpad administration group was significantly higher than that in the intravenous administration group $(\mathrm{P}<0.05)$. However, the administration of deuteporfin in the left rear footpad is limited in a clinical setting due to the inconvenience of the required operation, particularly for intraperitoneal organs, including the pancreas. In addition, the longer distribution-equilibrium time of deuteporfin in tissues associated with footpad administration may prolong the lucifugal or light-repelling time of the patients. Taken together, these results indicated that intravenous administration was the most appropriate method for deuteporfin, compared with administration via the left rear footpad.

Deuteporfin appeared to be selectively enriched in cancerous pancreatic lymph nodes, and it was therefore hypothesized that deuteporfin may be a potential tracer agent for the detection of lymphatic metastases.

To the best of our knowledge, the present study was the first to explore the potential use of deuteporfin as a tracer for identifying the lymphatic metastases of pancreatic cancer. Two administration routes were compared, and intravenous administration was found to be more practical than the local lymphatic route for human pancreatic cancer treatment, with 
the advantages of ease of administration and more rapid metabolism. The application of deuteporfin in the BxPC-3-LN5 human pancreatic cancer xenograft model, clearly exhibited the potential of deuteporfin as a tracer agent in the lymphatic metastases model of a pancreatic cancer cell line.

\section{Acknowledgements}

The present study was financially supported by the Shanghai Municipal Health Bureau (no. 2010Y081), the Shanghai Medical College of Fudan University (no. 10L-10) and the National Science Foundation of China (no. 81201896).

\section{References}

1. Saleem A, Jackson A, Mukherjee S, Stones N, Crosby T, Tait D and Price P; ACORRN UK Pancreatic Radiotherapy Group: Radiotherapy in the management of unresectable locally advanced pancreatic cancer: A survey of the current UK practice of clinical oncologists. Clin Oncol (R Coll Radiol) 22: 257-260, 2010.

2. Siegel R, Naishadham D and Jemal A: Cancer statistics, 2013. CA Cancer J Clin 63: 11-30, 2013.

3. Hidalgo M: Pancreatic cancer. N Engl J Med 362: 1605-1617, 2010.

4. Braat H, Bruno M, Kuipers EJ and Peppelenbosch MP: Pancreatic cancer: Promise for personalized medicine. Cancer Lett 318: 1-8, 2012.

5. Chan A, Diamandis EP and Blasutig IM: Strategies for discovering novel pancreatic cancer biomarkers. J Proteomics 81 : $126-134,2013$

6. McAllaster JD and Cohen MS: Role of the lymphatics in cancer metastasis and chemotherapy applications. Adv Drug Deliv Rev 63: 867-875, 2011.

7. Yang F, Jin C, Subedi S, Lee CL, Wang Q, Jiang Y, Li J, Di Y and Fu D: Emerging inorganic nanomaterials for pancreatic cancer diagnosis and treatment. Cancer Treat Rev 38: 566-579, 2012.

8. Russell M and Anzai Y: Ultrasmall superparamagnetic iron oxide enhanced MR imaging for lymph node metastases. Radiography 13: e73-e84, 2007.

9. Abdollahi A, Jangjoo A, Dabbagh Kakhki VR, Rasoul Zakavi S, Memar B, Naser Forghani M, Mehrabibahar M and Sadeghi R: Factors affecting sentinel lymph node detection failure in breast cancer patients using intradermal injection of the tracer. Rev Esp Med Nucl 29: 73-77, 2010.

10. Varghese P, Mostafa A, Abdel-Rahman AT, Akberali S, Gattuso J, Canizales A, Wells CA and Carpenter R: Methylene blue dye versus combined dye-radioactive tracer technique for sentinel lymph node localization in early breast cancer. Eur J Surg Oncol 33: 147-152, 2007.

11. Ogasawara Y, Yoshitomi S, Sato S and Doihara H: Clinical significance of preoperative lymphoscintigraphy for sentinel lymph node biopsy in breast cancer. J Surg Res 148: 191-196, 2008.

12. Noh YW, Kong SH, Choi DY, et al: Near-infrared emitting polymer nanogels for efficient sentinel lymph node mapping. ACS Nano 6: 7820-7831, 2012.

13. Agostinis P, Berg K, Cengel KA, Foster TH, Girotti AW, Gollnick SO, Hahn SM, Hamblin MR, Juzeniene A, Kessel D, et al: Photodynamic therapy of cancer: An update. CA Cancer J Clin 61: 250-281, 2011.
14. Dolmans DE, Fukumura D and Jain RK: Photodynamic therapy for cancer. Nat Rev Cancer 3: 380-387, 2003.

15. Lovell JF, Liu TW, Chen J and Zheng G: Activatable photosensitizers for imaging and therapy. Chem Rev 110: 2839-2857, 2010.

16. Issa MC and Manela-Azulay M: Photodynamic therapy: A review of the literature and image documentation. An Bras Dermatol 85: 501-511, 2010 (In English, Portuguese).

17. Nishioka NS: Drug, light, and oxygen: A dynamic combination in the clinic. Gastroenterology 114: 604-606, 1998.

18. Fan BG and Andrén-Sandberg A: Photodynamic therapy for pancreatic cancer. Pancreas 34: 385-389, 2007.

19. Brown SB, Brown EA and Walker I: The present and future role of photodynamic therapy in cancer treatment. Lancet Oncol 5: 497-508, 2004

20. Celli JP, Spring BQ, Rizvi I, Evans CL, Samkoe KS, Verma S, Pogue BW and Hasan T: Imaging and photodynamic therapy: Mechanisms, monitoring, and optimization. Chem Rev 110: 2795-2838, 2010

21. Abd-Elgaliel WR, Cruz-Monserrate Z, Wang H, Logsdon CD and Tung $\mathrm{CH}$ : Pancreatic cancer-associated Cathepsin E as a drug activator. J Control Release 167: 221-227, 2013.

22. Anand S, Ortel BJ, Pereira SP, Hasan T and Maytin EV: Biomodulatory approaches to photodynamic therapy for solid tumors. Cancer Lett 326: 8-16, 2012.

23. Pawlik TM, Gleisner AL, Cameron JL, et al: Prognostic relevance of lymph node ratio following pancreaticoduodenectomy for pancreatic cancer. Surgery 141: 610-618, 2007.

24. Ni X, Yang J and Li M: Imaging-guided curative surgical resection of pancreatic cancer in a xenograft mouse model. Cancer Lett 324: 179-185, 2012.

25. Fidler IJ: Rationale and methods for the use of nude mice to study the biology and therapy of human cancer metastasis. Cancer Metastasis Rev 5: 29-49, 1986.

26. Tamada Y, Aoki D, Nozawa S and Irimura T: Model for paraaortic lymph node metastasis produced by orthotopic implantation of ovarian carcinoma cells in athymic nude mice. Eur J Cancer 40: 158-163, 2004.

27. Schroder T, Chen IW, Sperling M, Bell RH Jr, Brackett K and Joffe SN: Hematoporphyrin derivative uptake and photodynamic therapy in pancreatic carcinoma. J Surg Oncol 38: 4-9, 1988.

28. Barrett AJ, Kennedy JC, Jones RA, Nadeau P and Pottier RH: The effect of tissue and cellular $\mathrm{pH}$ on the selective biodistribution of porphyrin-type photochemotherapeutic agents: A volumetric titration study. J Photochem Photobiol B 6: 309-323, 1990.

29. Dougherty TJ, Gomer CJ, Henderson BW, Jori G, Kessel D, Korbelik M, Moan J and Peng Q: Photodynamic therapy. J Natl Cancer Inst 90: 889-905, 1998.

30. Alexiades-Armenakas M: Laser-mediated photodynamic therapy. Clin Dermatol 24: 16-25, 2006.

31. Kilarski WW, Muchowicz A, Wachowska M, Mężyk-Kopeć R, Golab J, Swartz MA and Nowak-Sliwinska P: Optimization and regeneration kinetics of lymphatic-specific photodynamic therapy in the mouse dermis. Angiogenesis 17: 347-357, 2013.

32. Taylor MJ, Hoerauf A and Bockarie M: Lymphatic filariasis and onchocerciasis. Lancet 376: 1175-1185, 2010.

33. Padera TP, Kadambi A, di Tomaso E, Carreira CM, Brown EB, Boucher Y, Choi NC, Mathisen D, Wain J, Mark EJ, et al: Lymphatic metastasis in the absence of functional intratumor lymphatics. Science 296: 1883-1886, 2002.

34. Wang R, Hao H, Wang G, Xie H, Xu M, Wang W, He H and Li X: Pharmacokinetics, tissue distribution and excretion of a new photodynamic drug deuxemether. J Photochem Photobiol B 90: 179-186, 2008. 